\title{
Value Driven Conceptual Design of Unmanned Air System for Defense Applications
}

\author{
Evangelos Papageorgiou ${ }^{1}$, Hakki Eres ${ }^{2}$ and James Scanlan ${ }^{3}$ \\ Computational Engineering and Design Research Group, Faculty of Engineering and the Environment, University \\ of Southampton, Southampton, SO16 7QF, UK
}

\begin{abstract}
The work presented concerns the development of a value driven conceptual design assessment framework for a small Unmanned Air System (UAS) to be utilized in a defense application. In the field of Multi-Disciplinary Design Optimization, most recent systematic search has been devoted to fixed topology parametric geometries, pertaining to a single concept, with very little stress put on the optimization of variable topologies describing alternative design concepts. The search is conducted in a highly novel manner, generating a broad range of combinations of UAS configurations and geometries by systematically searching alternative concepts and design configurations through the parameterization of the aircraft geometric topologies. Moreover, the "value" of proposed solutions is assessed in an objective way both from performance and economic perspectives, while the optimal solution is identified after relaxing all of the design constraints as advocated by value driven design philosophy. During the multi-criteria decision analysis, the quantification/conversion of the linguistic preferences of the user between the various attributes to numerical values has disclosed some deficiencies introduced by the unjustifiable numerical scales used in the Analytic Hierarchy Process (AHP). This problem is resolved by a novel value model synthesizing the AHP assessment methodologies with multi-attribute value-focused analysis.
\end{abstract}

\section{Nomenclature}

$\begin{array}{ll}\text { AHP } & =\text { Analytic Hierarchy Process } \\ K & =\text { Scaling Factor } \\ \text { MAUT } & =\text { Multi-Attribute Utility Theory } \\ \text { MDO } & =\text { Multidisciplinary Design Optimization } \\ n & =\text { Design Attribute Norm } \\ \tau & =\text { Design Attribute Tolerance } \\ U & =\text { Utility Function } \\ U A S & =\text { Unmanned Air System } \\ V D D & =\text { Value Driven Design } \\ V & =\text { Value Function } \\ X & =\text { Design Attribute }\end{array}$

\section{Introduction}

CCORDING to Wiese', the successful exploration of the design space in the generic engineering design
oneeds to be a) 'systematic', in the way all potential solutions are proposed and evaluated against the main
objectives; b) 'iterative', using both simulation and prototyping to assess them; and c) 'multidisciplinary',

1 Ph.D. student, Faculty of Engineering and the Environment, Southampton Boldrewood Innovation Campus, Building 176, University of Southampton, Burgess Road, Southampton, SO16 7QF, UK, Tel.:0044 7581812956, email: ep1v12@ soton.ac.uk (corresponding author), AIAA Member Grade: Student.

${ }^{2}$ Senior Research Fellow, Faculty of Engineering and the Environment, Southampton Boldrewood Innovation Campus, Building 176, Room 5025, University of Southampton, Burgess Road, Southampton, SO16 7QF, UK. Tel.:0044 2380598368, email: hakki.eres@ soton.ac.uk, AIAA Member Grade: Regular.

${ }^{3}$ Professor, Faculty of Engineering and the Environment, Southampton Boldrewood Innovation Campus, Building 176, Room 5027, University of Southampton, Burgess Road, Southampton, SO16 7QF, UK. 
encompassing all important considerations across different disciplines. All key system aspects of all lifecycle stages of the designed system, from development and production to its disposal need to be addressed when studying the system, its components and their interactions with the wider environment.

The search for an optimum arrangement should not be limited only to traditional solutions, currently or commonly used. Following the Value Driven Design (VDD) approach, introduced by Collopy and Hollingsworth ${ }^{2}$, the design of a small Unmanned Air System (UAS) for defense use is distinguished/developed after removing any design constraints and extensively exploring the design space to identify design alternatives based on decision opportunities reflecting the user's values. The value driven conceptual design assessment framework assesses the "value" of proposed systems solutions by employing the multiple criteria decision analysis approach to address all of the economic and non-economic needs of the user and to identify the value-enhancing design(s).

This is research directed towards the following considerations:

- Employment of a wide range of UAS platforms.

- Quick and efficient conceptual design exploration.

- System definition in a terminology and language relevant to the designer, easily amended and replaceable for higher accuracy.

- Unit acquisition costing system and operational scenarios defined, to obtain accurate estimates of life cycle cost.

- Value/Utility assessment and refinement analytically derived from needs/requirements and all relevant design attributes.

\section{Value Driven Engineering Design}

Through the iterative engineering design process of Fig. 1, several potential design solutions, with different characteristics are generated and evaluated against the primary objectives to select the best one, as proposed by

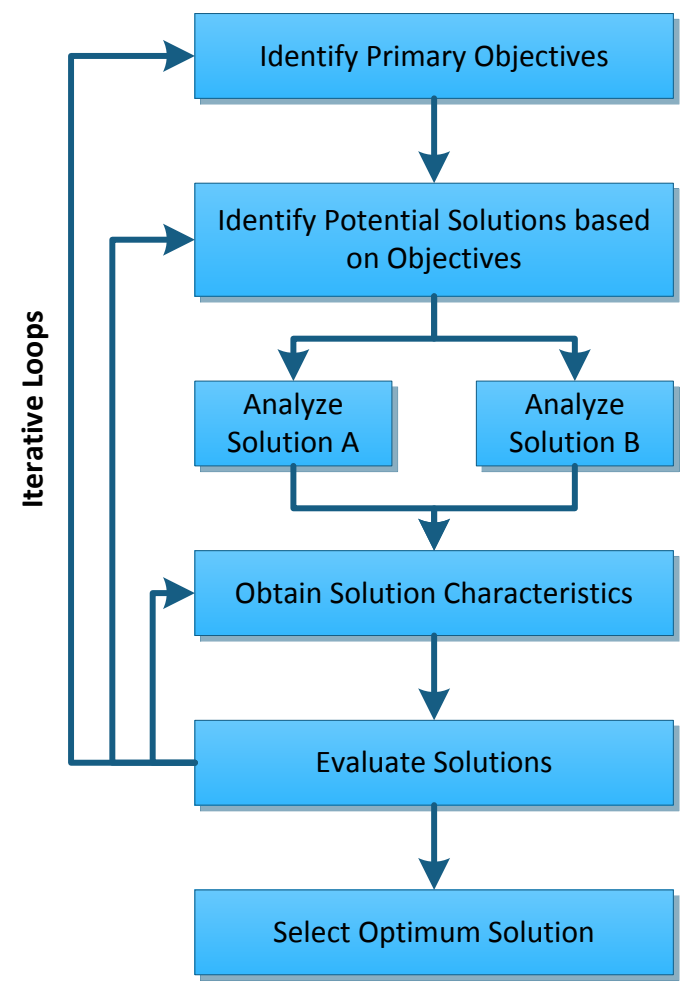
Hazelrigg ${ }^{3}$.

The first step in the engineering design process is to define the stakeholders' needs/requirements covering the complete lifecycle of the product. Identifying and structuring these needs as objectives articulates the values of the user, directing both the collection of information and decision making, during the generation and evaluation of potential alternative solutions respectively, as Keeney ${ }^{4}$ describes. In the proposed value modelling framework, the user of the UAS was assumed as the only stakeholder and the hierarchy of their objectives/attributes is presented in Fig. 2.

Figure 1 Engineering Design Process ${ }^{3}$ 


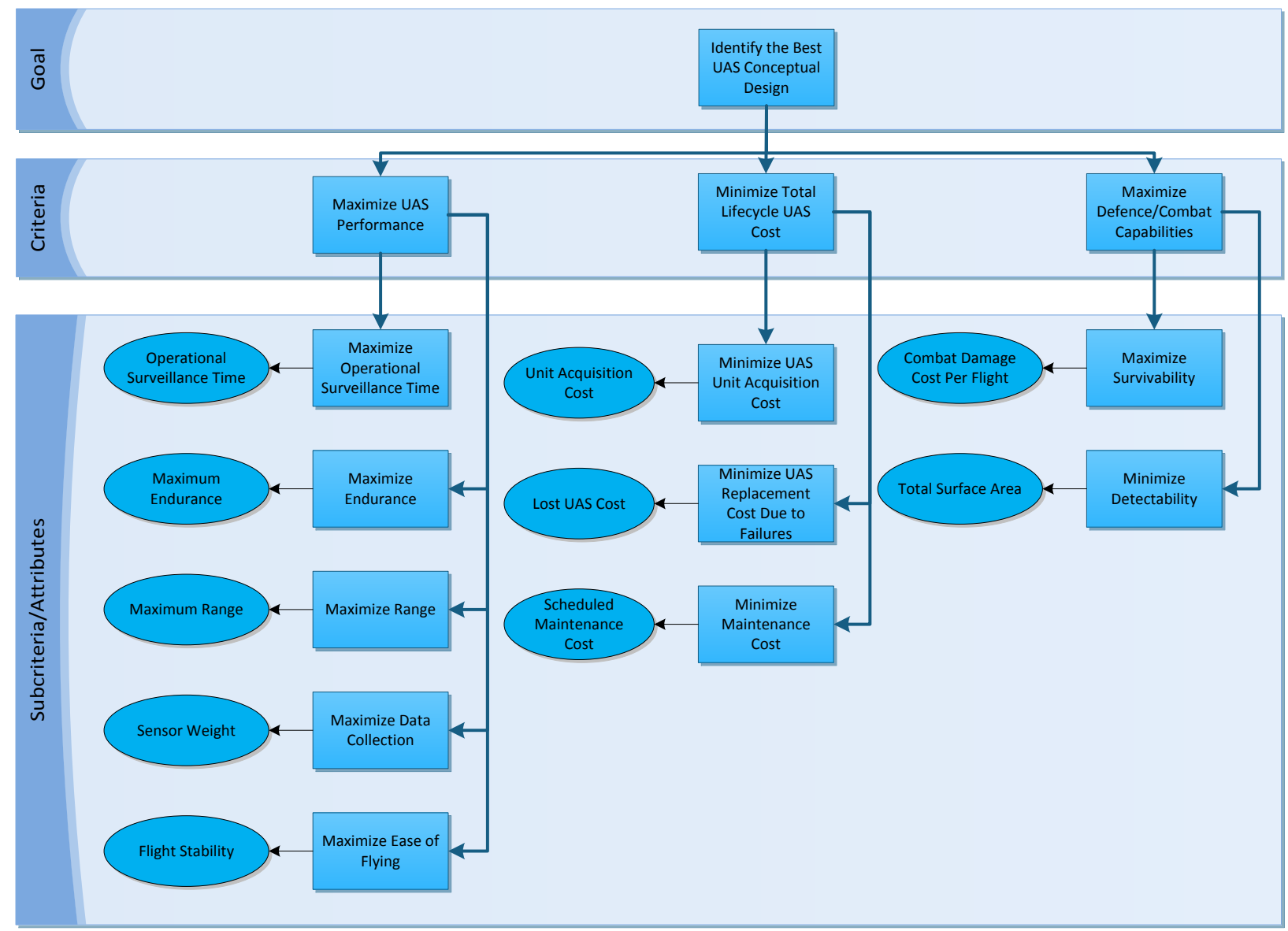

Figure 2 UAS Objectives/Attributes Hierarchy

The systems engineering approach introduced in the engineering design process by Hazelrigg ${ }^{5}$, is still the dominant integrating framework for engineering design. However, Collopy and Hollingsworth ${ }^{2}$ proposed a value centered optimization process to address delays and cost overruns experienced in engineering design, following the same approach of breaking the system to subsystems and components; but utilizing the system value model/objective function instead of the design requirements being flown down to the subsystems and components.

The UAS conceptual VDD framework is presented in Fig. 3. The chosen design variables are adjusted to generate feasible design points in the Define phase of the cycle. Extensive system attributes are calculated in the Analyze phase, such as specific fuel consumption, range, endurance, lifecycle cost, etc. And, they are used as inputs to the value model during the Evaluate phase. The process carries on, in the Search phase through an optimization algorithm or generation of more design points. 


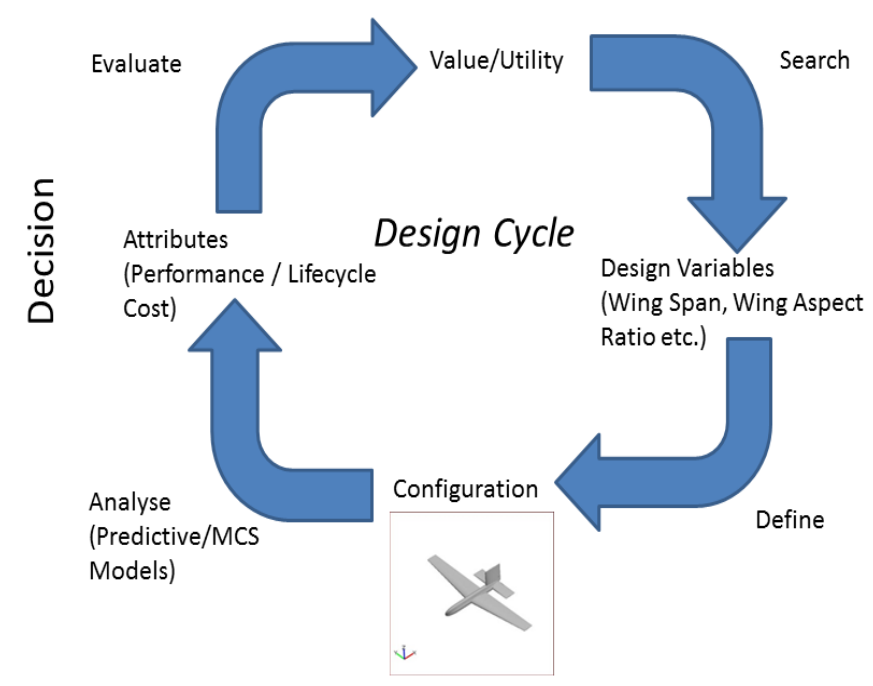

Figure 3 Value Driven Design Cycle

5. The longitudinal position of the stabilizer (forward or aft).

6. Its vertical position, relative to the fuselage (conventional horizontal tail or T-shape tail).

7. The existence and number of vertical fins.

8. The position of the propeller (forward or aft).

9. And finally the selection (or not) of all moving control surfaces generate more aircraft geometries.

These selections are presented in Fig. 4. Hence, a multitude of basic aircraft geometries is generated, described by a hierarchical coding composed as a series of 0 's and 1's, which were used as inputs into the appropriate numerical models defining the aircraft and estimating its relevant attributes. For example, an aircraft with monocoque fuselage, horizontal tail, one vertical fin, a pusher propeller and no all moving control surfaces would be coded as 111110110, a flying wing with a pusher propeller as 100000010 and an aircraft with a twin boom fuselage, inverted V-shape tail, pull propeller as 110011000 . This representation of a large number of aircraft geometric topologies allows for the shape definition to be input in the design models, which are then scaled through the use of appropriate design variables, such as wing span, wing aspect ratio, horizontal aspect ratio etc. Thus, the designer considers numerous advantages and disadvantages of each design choice and explores several UAS configurations, identifying a different optimal design depending on his/her preferences and priorities. 


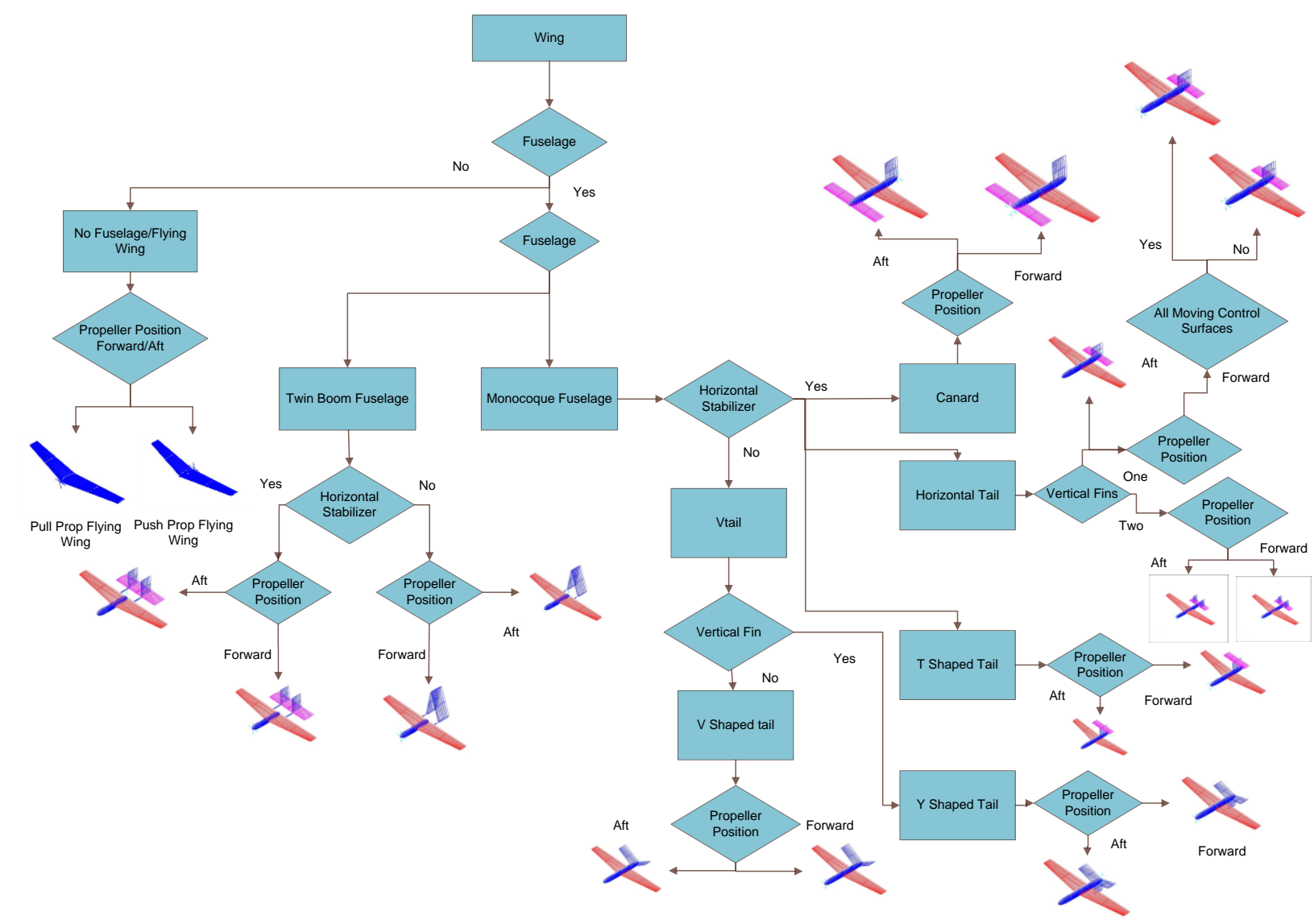

Figure 4 Aircraft Geometries Generation

\section{B. UAS Design Generation and Analysis}

The aircraft design generation is performed in four steps:

1. Aircraft sizing, including structural analysis for basic structural components, drag calculations, engine performance and propulsion analysis, weight and balance calculations and aerodynamic analysis.

2. The acquisition cost analysis, using the design parameters, product definition and geometry, along with the material and labor cost rates to calculate the UAS unit and total program cost.

3. The operational simulation analysis model, calculating the maintenance lifecycle cost and losses due to unreliability.

4. The simulation survivability analysis model, providing estimates of the combat damage cost and associated UAS battle losses.

In lifecycle cost modelling, it was assumed that the failures due to lack of reliability and scheduled maintenance, along with the survivability related combat damages are the governing factors defining lifecycle cost and operational missions' availability of the UAS fleet. These failures due to unreliability of critical subsystems/components are modelled by Weibull distributions and through Monte Carlo simulations lifecycle cost and survivability related damage cost and uncertainties are estimated.

The chosen design variables for the design alternatives generation are the wing aspect ratio, wing taper ratio, wing span, wing position, horizontal tail aspect ratio, fin aspect ratio, canard aspect ratio, for the canard configuration, battery capacity, since electric propulsion is assumed, and component reliability used for the lifecycle cost calculations, as the probability for any component to remain operative until its scheduled replacement.

\section{Value Modelling}

Unless the goodness of any design can be modelled by a single attribute, usually of monetary type, an array of parameters of incommensurable units, reflecting performance, environmental and other even intangible concerns, 
depicts its overall worth. A value model assessing the preferences of the stakeholder(s) objectively, with inputs of these parameters, serves as the corresponding objective function.

\section{A. Multi-Attribute Value Model}

Assuming preferential independence among the attributes, as defined by Keeney and Raiffa ${ }^{6}$, a novel additive multi-attribute value model was developed, with scaling factors $K_{i}$ representing the 'weighting importance' of each attribute within the set/subset it belongs and $V_{i}$ the corresponding value functions of the associated attributes $X_{i}$ :

$$
V\left(X_{1}, X_{2}, \ldots, X_{n}\right)=\sum_{i=1}^{n} K_{i} \cdot V_{i}\left(X_{i}\right)
$$

Norm is defined as 'an average level of development or achievement, something that is usual or expected, an authoritative standard, usually derived from the average or median achievement of a large group ${ }^{77}$. The assignment of norms of average levels of expectations with respect to the attributes by the user is the basis of this novel multiattribute norm value model, used both for the scaling constants $K_{i}$ and value functions $V_{i}$ assessments. Thus, as Keeney ${ }^{4}$ advocates, the alternative-focused process of selecting the best from what is readily available, is converted to a value-focused process of identifying needs, attributes and values of these attributes that give the user a 'neutral' response, a $50 \%$ satisfaction level, as described by Eres et al. ${ }^{8}$ in the Concept Design Analysis (CODA) methodology. The major advantage of this value model is that it is an efficient and operational way to evaluate each design point, during the conceptual phase when only basic needs and vague requirements are known and the set of design alternatives is not finalized, with the minimum interaction with the user. Moreover, the objectivity of the evaluation is maintained by capturing the user's preferences, with criteria independent of information, other data available or the proposed alternative solutions.

\section{Value Functions}

Usually, most value functions use the a posteriori assignment of values to specific attribute levels, such as the best and worst obtained level, to perform some normalization. However, in this value model before the conceptual design space exploration starts and subject to the technology readiness level assumed, a priori the user provides some reference values for attributes that would give a 'neutral' response/satisfaction. These attribute levels are assigned a goodness value of $1 / 2$ for maximizing or minimizing value functions and 1 for the optimizing value functions, analogous to the Taguchi Loss Function.

The exponential function was selected, as a ready to use value function $V_{i}$ capable to capture the preferences of the user. This function can be adjusted for maximizing, minimizing or optimizing behavior towards a specific target value of each attribute, and can also be adjusted in shape (as concave or convex) reflecting the marginal evaluation of the user with respect to each attribute, i.e. willing to sacrifice more and more (convex) or less and less (concave) in terms of other attributes for the same positive increment of this attribute as its values increase.

Depending on the previous selections, the ready to use value functions, presented in Table 1, are employed. In these equations, $X$ is the input attribute value for any design, $n$ is the assigned norm of this design attribute while $V_{i n}$ and $V_{f}$ are the initial and final values of the value functions (set accordingly, depending on its type). 
Table 1Value Functions

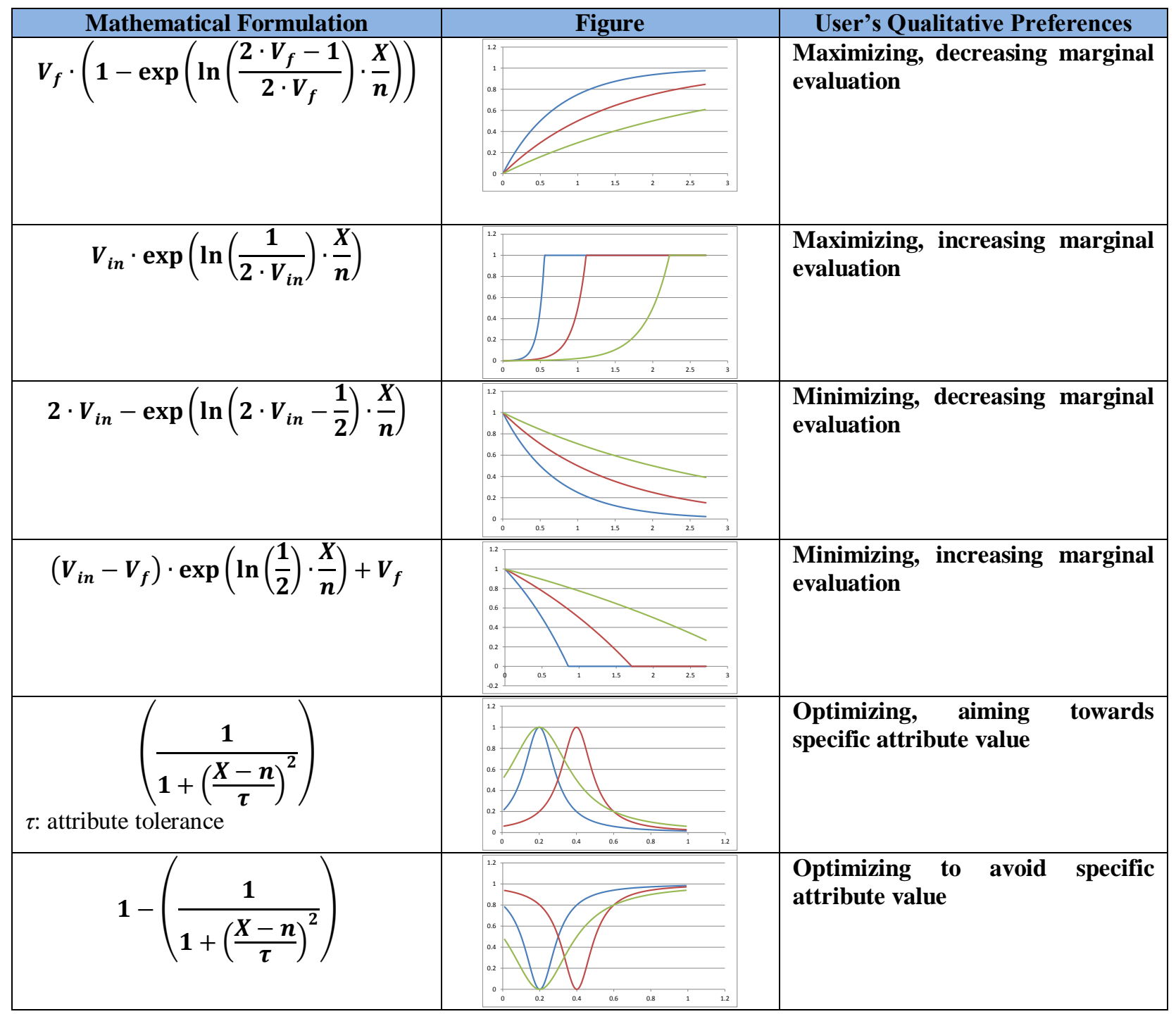

2. Weighting Factors Assessment

For the assessment of the weighting importance of each attribute, the AHP method was first employed to perform pairwise comparisons between the attributes and to assess the consistency of the answers provided by the stakeholder. However, the construction of the AHP matrix was based on the ambiguous question: 'How much better/more important is attribute/alternative $A_{i}$ than $A_{j}$ ?', assessing the ratio scaled strength of preference. It has been found that this unjustifiable selection of numerical scale, such as those presented by Elliot ${ }^{9}$, converting the linguistic response to the above question to ratio scaled numerical values, greatly affected the identification of optimal design. These scales, expressing the user's psychological perception of strength of preference increments among the various attributes; as presented in Table 2 for integer and power scales, quantify exactly the same linguistic responses to different numerical values. For instance the verbal response: ' $A_{i}$ is much more important to attribute $A_{j}$ ' is converted to a numerical value of 7 with integer scale and to a value of 5.2 with power scale, with the integer scale favoring more the most important to the user attributes and weighing less the least important ones. 


\begin{tabular}{|l|l|l|l|}
\hline Definition & $\begin{array}{l}\text { Integer } \\
\text { Scale }\end{array}$ & $\begin{array}{l}\text { Power } \\
\text { Scale }\end{array}$ & Explanation \\
\hline Equal Importance & 1 & 1 & Two factors contribute equally \\
\hline Somewhat more important & 3 & 1.73 & Slightly favor one over the other \\
\hline Much more important & 5 & 3 & Strongly favor one over the other \\
\hline Very much more important & 7 & 5.2 & Very strongly favor one over the other \\
\hline Absolute more important & 9 & 9 & Highest possible validity of favoring one over the other \\
\hline
\end{tabular}

Due to the absence of justified criteria for selecting a particular scale converting the linguistic answer/stimuli to a numerical value/response, the distribution of weights and level of consistency obtained by different scales can be used in AHP to select the best one. In order to study the distribution of weights for the integer, balanced and power scales, all possible combinations of weighting scales were obtained and presented (in this order) in Fig. 5, for a nine degree preference scheme between three attributes, similar to Fig. 1 of Elliot ${ }^{9}$. In this figure, the coordinates of each point are the weights of the two attributes, whereas the third can be computed since their sum is always equal to unity. Thus, the three scales can be compared in terms of the number of distributed points and existence of sparse
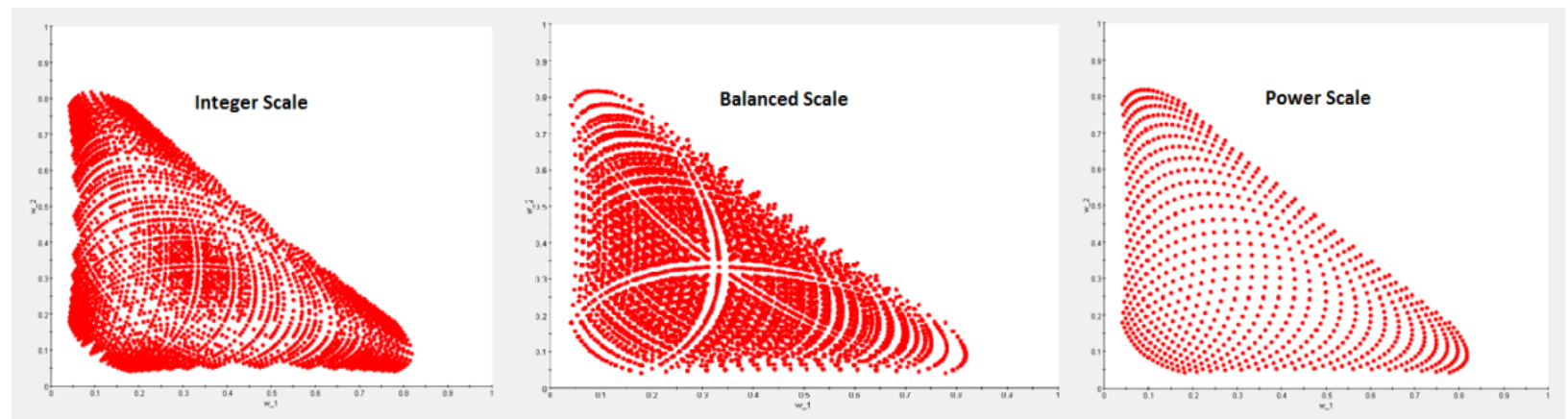

Figure 5 AHP Numerical Scales Weight Distribution

regions, in order to select the scale with the highest number of points and the least sparse regions.

In Fig. 5, the following are noticed:

- The integer scale gives a high number of points in the most extreme values of weights as expected, considering the distribution of values of the integer scale: $1,2,3,4,5,6,7,8,9$, that weighs more the most important to the user attributes and less the least important ones, as compared to the distribution of values of the balanced scale: $1,11 / 9,4 / 3,13 / 7,7 / 3,3,4,17 / 3,9$ and the distribution of values of the power scale: $1,1.316,1.732,2.28,3,3.948,5.196,6.84,9$. Therefore, the balanced and power scales are more evenly distributed than the integer scale.

- Comparing the sparse regions and clustering obtained with the three scales; the power scale fails to cover a larger area in the graph than the integer and balanced scales which produce a definitely higher number of points and less clustering. This observation is not in agreement with Elliot ${ }^{9}$ who notices larger sparse regions and clustering of the weights in integer and balanced scales and identifies the power scale as the most preferable.

Furthermore, to explore the use of different scales in AHP, and based on the value model using the two scales, integer and power scale, as defined in Table 2, a multi-disciplinary optimization was performed. It was found that exactly the same verbal responses/preferences provided by the user, when converted to numerical weights through different AHP scales produced different optimal aircraft designs. Hence, through the use of integer scale, a V-shape tail, push propeller, conventional fuselage with a wing span $1.5 \mathrm{~m}$ aircraft was identified as optimal, while through the power scale, an aircraft of T-shape tail, pull propeller, conventional fuselage with a wing span of $1.25 \mathrm{~m}$.

The problem of converting verbal preference responses between attributes to numerical values through the use of some unjustifiable scale in AHP for the calculation of weighting factors can be tackled if instead of performing pairwise comparisons between abstract attributes, the user is forced to compare specific value differences of these 
attributes. To assess the strength of preferences (value differences) between alternatives, based on the theory of measurable multi-attribute value functions presented by $\mathrm{Dyer}^{10}$, apart from preference independence, weak difference independence is additionally assumed, i.e. that the order of preference differences between pairs of each attribute is independent of the levels of the other attributes.

The attribute norms already used for the value functions assessment are again employed in a direct rating approach to compute the scaling constants by assessing the relative importance of:

- The preference difference between a design with attribute $x_{i}$ at the norm, $x_{i}^{1 / 2}$, and all other attributes $\bar{x}_{i}$ at their worst value, $\bar{x}_{i}^{o}$, and the design with attribute $x_{i}$ at the worst or best (value of 0 or 1 , depending on the form of value function), $x_{i}^{*, o}$, and all other attributes $\bar{x}_{i}$ at their worst value, $\bar{x}_{i}^{o}$.

- And the preference difference between a design with attribute $x_{j}$ at the norm, $x_{j}^{1 / 2}$, and all other attributes $\bar{x}_{j}$ at their worst value, $\bar{x}_{j}^{o}$, and the design with attribute $x_{j}$ at the worst or best, $x_{j}^{*, o}$, and all other attributes $\bar{x}_{j}$ at their worst value, $\bar{x}_{j}^{o}$.

Through this relative importance assessment of changes to the norm value of any two attributes, the use of any numerical scale can be avoided. Each comparison is represented by Eq. (8), used to assess the ratio of the weighting factors:

$$
\frac{k_{i \cdot} \cdot\left(U_{i}\left(x_{i}^{0.5}\right)-U_{i}\left(x_{i}^{0, *}\right)\right)}{k_{j} \cdot\left(U_{j}\left(x_{j}^{0.5}\right)-U_{j}\left(x_{j}^{0, *}\right)\right)}=\frac{k_{i}}{k_{j}}=c_{i j}
$$

In Table 3, instead of comparing abstract attributes, each cell is the ratio of relative importance/preference of a change to the norm value of row attribute to the change to the norm value of column attribute (from their lowest/highest values). Following the methodology of AHP, several pairwise comparisons are performed, not only to compute the values of the weighting factors, but also to assess the consistency of the answers provided by the

Table 3AHP Weighting Factors Assessment

\begin{tabular}{|c|c|c|c|c|c|c|c|c|c|c|c|c|c|c|}
\hline User Needs & 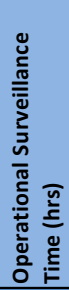 & 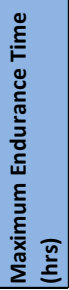 & 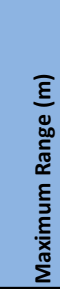 & 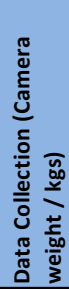 & 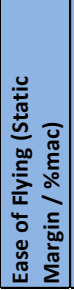 & 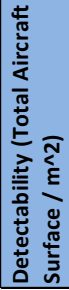 & 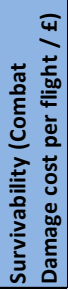 & 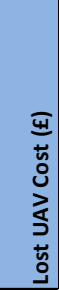 & 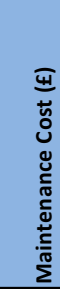 & 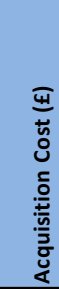 & 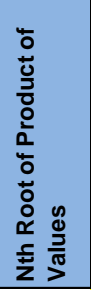 & 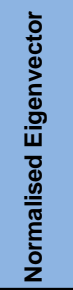 & 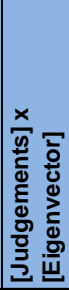 & 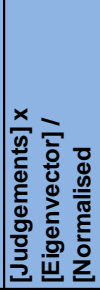 \\
\hline Operational Surveillance Time (hrs) & 1.000 & 1.333 & 1.333 & 2.000 & 2.000 & 4.000 & 4.000 & 2.000 & 2.000 & 1.333 & 1.898041 & $17.64 \%$ & 1.7767 & 10.06992 \\
\hline Maximum Endurance Time (hrs) & 0.750 & 1.000 & 1.000 & 1.333 & 1.333 & 2.000 & 4.000 & 1.333 & 1.333 & 1.333 & 1.381289 & $12.84 \%$ & 1.292 & 10.06194 \\
\hline Maximum Range (m) & 0.750 & 1.000 & 1.000 & 1.333 & 1.333 & 2.000 & 4.000 & 1.333 & 1.333 & 1.333 & 1.381289 & $12.84 \%$ & 1.292 & 10.06194 \\
\hline Data Collection (Camera weight / kgs) & 0.500 & 0.750 & 0.750 & 1.000 & 1.000 & 1.333 & 2.000 & 1.333 & 1.333 & 0.800 & 1.006475 & $9.36 \%$ & 0.9416 & 10.06436 \\
\hline Ease of Flying (Static Margin / \%mac) & 0.500 & 0.750 & 0.750 & 1.000 & 1.000 & 1.333 & 2.000 & 1.333 & 1.333 & 0.800 & 1.006475 & $9.36 \%$ & 0.9416 & 10.06436 \\
\hline Detectability (Total Aircraft Surface / $\mathbf{m}^{\wedge} \mathbf{2}$ ) & 0.250 & 0.500 & 0.500 & 0.750 & 0.750 & 1.000 & 1.333 & 0.667 & 0.667 & 0.500 & 0.633538 & $5.89 \%$ & 0.594 & 10.08553 \\
\hline Survivability (Combat Damage cost per flight / f) & 0.250 & 0.250 & 0.250 & 0.500 & 0.500 & 0.750 & 1.000 & 0.500 & 0.500 & 0.333 & 0.435275 & $4.05 \%$ & 0.4081 & 10.08484 \\
\hline Lost UAV Cost (f) & 0.500 & 0.750 & 0.750 & 0.750 & 0.750 & 1.500 & 2.000 & 1.000 & 1.000 & 0.800 & 0.907701 & $8.44 \%$ & 0.8484 & 10.05479 \\
\hline Maintenance Cost (f) & 0.500 & 0.750 & 0.750 & 0.750 & 0.750 & 1.500 & 2.000 & 1.000 & 1.000 & 0.800 & 0.907701 & $8.44 \%$ & 0.8484 & 10.05479 \\
\hline Acquisition Cost (f) & 0.750 & 0.750 & 0.750 & 1.250 & 1.250 & 2.000 & 3.000 & 1.250 & 1.250 & 1.000 & 1.199769 & $11.15 \%$ & 1.1205 & 10.04658 \\
\hline 10 & & & & & & & & & & & 10.75755 & 1 & MEAN & 10.08553 \\
\hline & & & & & & & & & & & \multicolumn{3}{|c|}{ CONSISTENCY INDEX } & 0.009503 \\
\hline & & & & & & & & & & & \multicolumn{3}{|c|}{ RANDOMCI } & 0.553 \\
\hline & & & & & & & & & & & \multicolumn{3}{|c|}{ CONSISTENCY RATIO } & $1.72 \%$ \\
\hline
\end{tabular}

stakeholder.

The major advantage of this value model is that it is a very efficient and operational way to evaluate each design point, during the conceptual phase when basic needs and vague requirements are known, with the minimum interaction with the user. This multi-attribute value model, based on the quantitative assignment of neutral values of attributes as norms and the assessment of the user's preferences, allows for the objective evaluation of all design alternatives during the conceptual phase, independent of information. Moreover, the deficiency introduced by the unjustifiable selection of numerical scale used in AHP is resolved through the synthesis of AHP with the measurable multi-attribute value functions. 


\section{B. Multi-Attribute Utility Model}

Multi-attribute utility theory (MAUT) is another appropriate tool for dealing with problems when more than one attributes are required to address the multiple objectives. For the UAS design framework, the process followed and all questions posed are as described by Keeney and Raiffa ${ }^{6}$. Preferential independence and utility independence were assumed, reducing the model to a multiplicative one with $K$ and $K_{i}$ scaling factors, while $U_{i}$ is the utility function for each attribute $X_{i}$ :

$$
K \cdot U(X)+1=\prod_{i=1}^{n}\left[K \cdot K_{i} \cdot U_{i}\left(X_{i}\right)+1\right]
$$

Utility functions for all attributes were assessed through a series of certainty to lottery equivalents, after standard parametric families of utility functions were selected. The scaling factors of the multiplicative model were evaluated with independent equations, generated from certainty and probabilistic considerations. The utility model requires extensive interaction with the user to assess their risk attitude, which is not always possible, especially during the conceptual design phase. The decision maker has to be open-minded, interested and willing to think hard about consequences, when answering the preferences assessment questions, and if necessary, reconsider to achieve a consistent set of preferences. This multi-attribute utility model is far more complicated and elaborate than the value model, and can be used as a second and more thorough approach to the stakeholder's preferences and risk attitude against uncertainties.

\section{Value Driven Design Model Integration}

The workflow execution and integration environment Isight ${ }^{11}$ was chosen as the integration platform of all models because of its ability to execute simulation-based processes in a visual and flexible way, allowing use and control of various software components utilized in the design process and accelerating the design exploration and evaluation of the alternatives. The design space is explored without setting any constraints using the techniques of Design of Experiments (DoE), optimization and approximations, while post-processing tools perform sensitivity analysis and trade-off studies between design parameters and results.

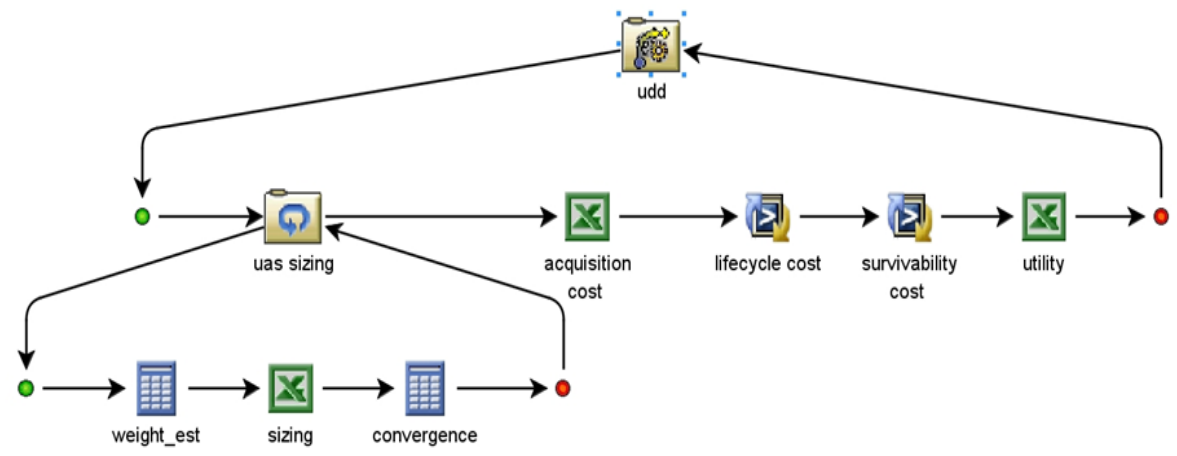

Figure 6 Isight UAS Design Framework
The Isight model used in the value driven UAS design framework is shown in Fig. 6.

The design space exploration aimed at maximizing value or utility index, depending on which value model was used, or alternatively optimizing some critical attribute, such as operational surveillance time, maximum endurance/maximum range achieved, or total lifecycle cost. The preferences/priorities of the user, as reflected in the value/utility models, are critical for the identification of the optimal design and can indeed provide different results. Thus, based on a user with 'balanced' priorities between performance (endurance, range) and lifecycle cost (acquisition and through-life) and a 'military' user, focusing mostly on maximizing survivability, minimizing UAS detectability and maximizing data collection capabilities, different aircraft optimal designs were obtained. For a user with 'balanced' priorities an aircraft with V-shaped tail and wing span of $1.5 \mathrm{~m}$ was identified as optimal, whereas for a 'military' user the UAS with T-shaped tail and a wing span of $1.25 \mathrm{~m}$ was the optimal configuration. 
Based on the user's specific preferences, dominant aircraft configurations/geometries maximizing value or utility index are identified, as in Fig. 7. The monocoque fuselage, V-shape tail pusher propeller configuration, followed by the monocoque fuselage, Y-shape tail, pusher propeller configuration and monocoque fuselage, $\mathrm{T}$-shape tail, pusher

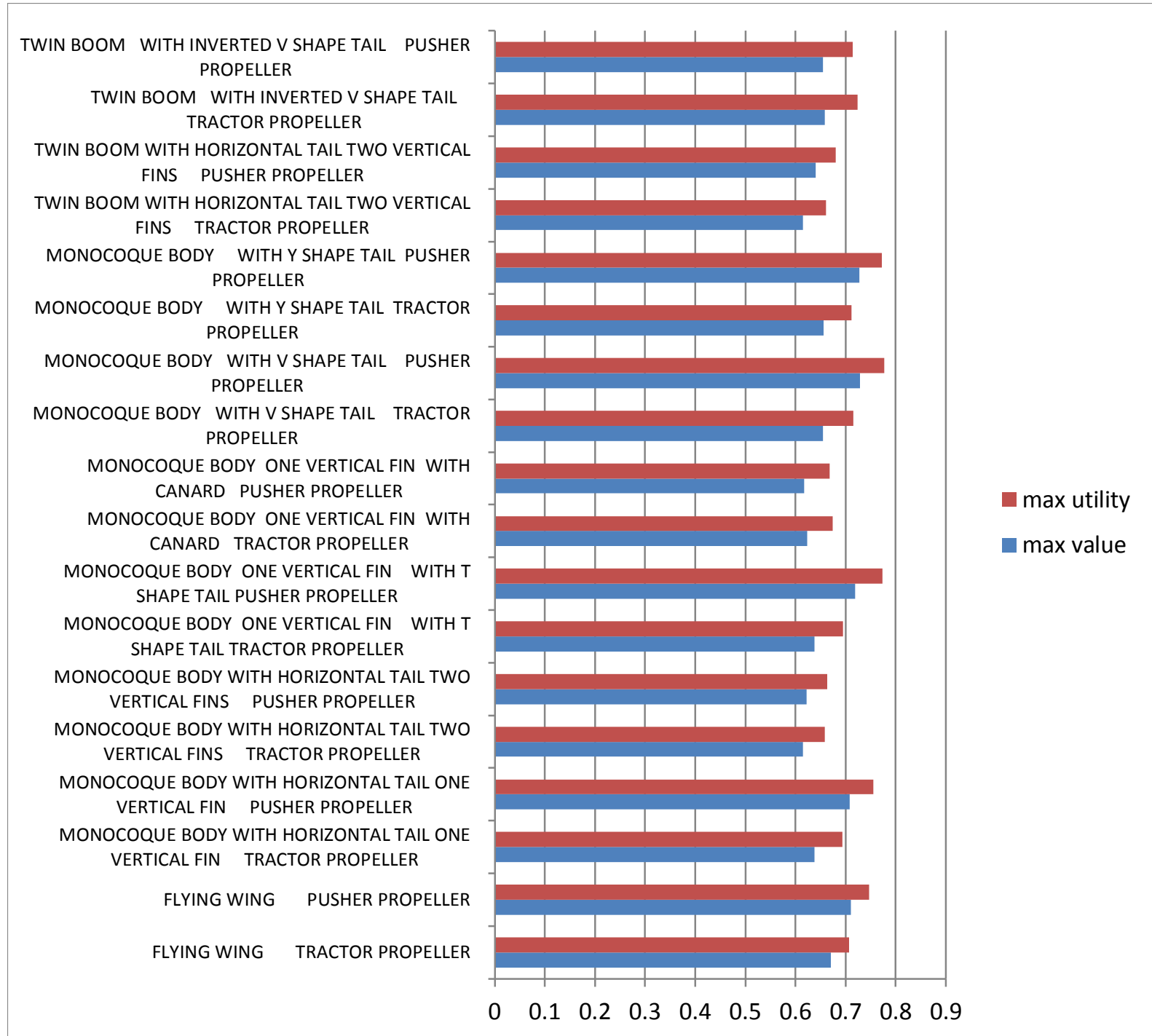

Figure 7 Comparison of Different Configurations

propeller configuration are dominating in terms of both value and utility indices.

Additionally, the optimum range of design variables was obtained, while surface plots demonstrate the effect of design variables or other parameters on the response, as in Fig. 8. Both value and utility models point to selecting the same ranges of design variables, such as a wing aspect ratio of around 12 , a wing span of $1.5 \mathrm{~m}$, a battery capacity of $9.5 \mathrm{Ahr}$, and an intermediate scheduled maintenance interval, while for some design variables with small influence, such as fin aspect ratio and horizontal tail aspect ratio, the optimal ranges are not clear. 


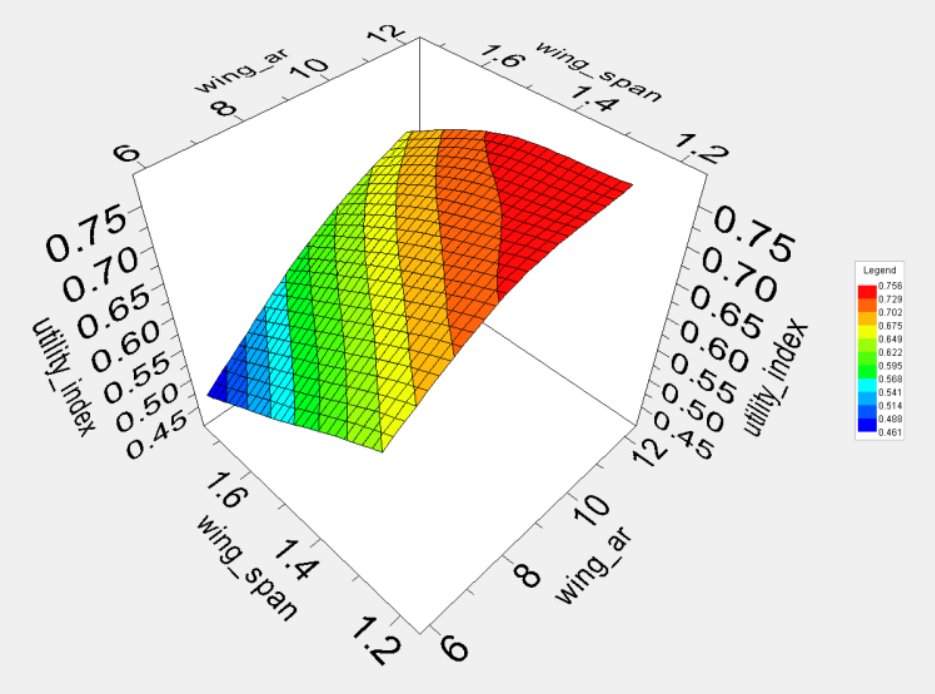

Figure 8 Surface Plot
Alternatively, optimizing for some critical aircraft attribute: the twin boom, inverted V-shape tail and tractor propeller configuration was identified as the dominant both for operational surveillance time when flying at design speed and total lifecycle cost. For maximum endurance, the flying wing with pusher propeller aircraft was found as expected to be the dominant one with wing aspect ratio of 12 and wing span of $1.25 \mathrm{~m}$, while for maximum range, a flying wing, pusher propeller configuration with wing aspect ratio of 6 and wing span of $1.25 \mathrm{~m}$ is the optimum one.

Moreover, the multi-attribute value model is validated by providing analogous results with the multi-attribute utility model and can address effectively the user's preferences. Both models identify the same aircraft configurations as dominant in terms of maximizing value or utility index, while in the surface plots, they capture similar effects of design variables on the response, value or utility.

\section{Discussion and Conclusion}

So far, the main objective of this research has been to create a framework that will apply the value driven design philosophy in the conceptual design phase of a defense UAS. The whole process of engineering design is converted into a multi-criteria decision making analysis while considering the following aspects:

- The conceptual VDD framework generates, defines and analyzes the aircraft design points, following a geometric topologies parameterization and calculates the value associated with each design based on the single stakeholder/user's needs or preferences.

- The VDD framework, performing an extensive design exploration and multidisciplinary design optimization, achieves automated design identification by relaxing all requirements and maximizing the value/utility objective function, as advocated by the VDD.

- The multi-attribute value model is successfully employed as a basic model during the conceptual phase design, capturing the stakeholder's needs as fundamental objectives defining the set of optimal design alternatives, framing and guiding engineering design towards the right direction. The problem of using an unjustifiable numerical scale in AHP to convert the linguistic answers to numerical values, is also tackled through this value model. For the calculation of weighting factors, the user instead of comparing abstract attributes, is forced to assess the strength of preferences (value differences) between design alternatives.

- The multi-attribute utility model has manifested the extensive interaction with the user, required to establish and assess their preferences concerning the consequences. This model could be used as a second and more thorough approach to the stakeholder's preferences and risk attitude against uncertainties, once the list of the design alternatives is finalized.

Current and future work includes the application/testing of this framework in a practical case, capturing customer preferences/needs and trade-offs between them, and assessing alternative aircraft configurations. Additionally, the views and needs of other than the user stakeholders, such as the UAS manufacturer or parts suppliers, need to be incorporated into the decision making framework.

\section{Acknowledgments}

This work has been conducted through the Southampton University Engineering Centre of Excellence. The authors would like to thank the Defense Science and Technology Laboratory (DSTL) for the funding and support of this project. 


\section{References}

1. Wiese, P.R., Engineering Design in the Multi-Discipline Era, A system approach. 2003, UK: Professional Engineering Publishing Limited.

2. Collopy, P.D. and P.M. Hollingsworth, Value-Driven Design. Journal of Aircraft, 2011. 48(3): p. 749-759.

3. Hazelrigg, G.A., Validation of Engineering Design Alternative Selection Methods. Journal of Engineering Optimization, 2003. 35(2): p. 103-120.

4. Keeney, R.L., Value-focused Thinking: a Path to Creative Decision Making. 1996, Cambridge, MA Harvard.

5. Hazelrigg, G.A., Systems Engineering: An Approach to Information-Based Design. 1996: Prentice-Hall, Upper Saddle River, NJ. pp. 158-161, 291-311.

6. Keeney, R.L., Raiffa, H., Decisions with Multiple Objectives. 1976, Cambridge, UK: Press Syndicate of the Cambridge University.

7. www.merriam-webster.com/dictionary.

8. Eres, M.H., Bertoni, M., Kossmann, M. and Scanlan, J.P., Mapping Customer Needs to Engineering Characteristics: An Aerospace Perspective for Conceptual Design. Journal of Engineering Design, 2014. 25(1-3): p. 64-87.

9. Elliot, M.A., Selecting Numerical Scales for Pairwise Comparisons. Reliability Engineering and System Safety, 2010. 95: p. 750-763.

10. Dyer, J.S., Sarin,R.K., Measurable Multiattribute Value Functions. Operations Research, 1979. 27(4): p. 810-822.

11. Isight \& the Simulia Execution Engine. Available from: http://www.3ds.com/products-services/simulia/portfolio/isightsimulia-execution-engine/overview/. 